\title{
The study on the landscape architectural design based on the Virtual Reality technology
}

\author{
Yan Long1, Rongke Jin2, Hong Xu3 \\ 1 College of Urban Construction,Wuhan University of Science and Technology, Wuhan \\ 2 School of Engineering and Architecture,Jiaxing University, Jiaxing \\ 3 College of Urban Construction,Wuhan University of Science and Technology, Wuhan \\ Corresponding E-mail:LO2000916@163.com
}

Keywords: Landscape architectural design; virtual

\begin{abstract}
The virtual reality technology is based on 3D graphics technology, combined the technology of multimedia, the main body of visual and sensor technology to create an immersive virtual world. The paper is based on the landscape architectural design technology of virtual reality, and builds the landscape architectural system with immersion virtual roaming and interactive virtual roaming, which can make students experience the incomparable sense of reality and immediacy, fully realize the pros and cons of the design works, and do some necessary modification to achieve the goal of optimizing designing.
\end{abstract}

\section{INTRODUCTION}

Virtual reality technology which introduced into building design allows designers or owners immersive experience the building built. Designers can be fully in accordance with their own ideas to construct the "virtual" room, and can transform the point of view anyway in room which understanding the pros and cons of building design, and constantly make changes until satisfied.Virtual reality technology allows the artistry of the building that can be emphasized and highlighted, and if the art features of the building are becoming pure, that is the space art. But in the building design process in the past, the space art is difficult to achieve. The space arts with virtual reality technology can be achieved in the field of landscape architectural design. Therefore, the paper proposed that the applied research of virtual reality technology is particularly necessary in the filed of landscape architectural design teaching. In this study, the author is virtual way task of landscape architectural design case, and the effect of classroom teaching cases were effective evaluation.The virtual simulation is also called the virtual reality technology and simulation technology, is to use a virtual system imitate another real system technology. Due to the development of computer technology, simulation technology is gradually, become the mathematical reasoning, scientific experiment after the third type of basic method of objective laws of nature in human knowledge, and is developed into human knowledge, the transformation and the creation of the objective world a generality, strategic technology. Virtual Reality technology, VR for short, is a comprehensive integrated in the $1980 \mathrm{~s}$ the rise of new technology, involved in computer graphics, human-computer interaction technology, sensor technology, artificial intelligence, etc. It is made up of computer hardware, software, and all kinds of sensor 3D information of artificial environment, a virtual environment, can realistically simulate the real world (or even non-existent) things and the environment, people are into the environment, feel "in its" immediately, and can be operated personally, naturally interact with virtual environment.

\section{The meaning of the three dimensional animation design}

With the further development of our country's digital electronic technology, in order to meet the requirement of the animation market, on the basis of the digital electronic technology to strengthen the use of the three dimensional animation technology. In the three dimensional animation designs, 
mainly is the combination of static and dynamic animation vivid visualization, movement to give more artistic appeal is the essence of animation design, animation is one of basis, is a form of sports art performance. The essence of animation design is expressed in the form of dynamic picture. In short, animation design includes two aspects, the dynamic and the combination of dynamic and static images. With the improvement of animation design technology, changed the traditional form of animation design, each picture will be displayed in the form of dynamic, in the true sense given the picture dynamic image and a sense of improve the expressive art of animation. With the gradually mature of the three dimensional animation design technology, digital art in the design of animation design using the same principle and traditional animation design, using computer technology and the related design technology, animation design and production, under the condition of the same basic principle, strengthen the reform and perfect technology, essentially improve the effect of the performance of animation design. Digital art the use of electronic technology for animation design provides a new development direction and forms, can meet the designers of the animation more expression of subjective thought, enhances the light shadow, screen, texture of animation design, sports and so on each aspect of the expression effect, animation design, a new leap. In the process of the three dimensional animation design, is using the digital electronic technology to create a real 3D scene, visual and vivid animation design more. In today's digital electronic technology becoming mature, many advanced sports theory was introduced into the three dimensional animation design software, 3D animation, in the design of these designer does not need to design each frame. Only need to design a good link between animations, meet the automatic conversion of animation, to form a dynamic picture. This technique effectively improves the design efficiency, and makes the picture of cohesion between natural, smooth, forming a sense of the whole dynamic picture. Through the three dimensional animation design software, the static picture designed first, and then using the kinematics theory, design a key turning point in the picture, finally from the images of the whole, the three dimensional animation effect.

\section{Landscape architectural design Image recognition and registration}

First make recognition objects, Vuforia AR SDK support local identification database and cloud identification database, these two methods have their own strengths, local database don't need network to support and complete recognition faster, but a limited number of objects can be identified and must be made in advance, in contrast, the cloud identification may support more than one million objects, but this method must rely on the high-speed internet connection, considering the conditions of ship using, decided to use local identification database, we need to upload the images or markers which need to associate with augmented contents to Qualcomm "Target Manager" and generate local identification data set.

Then put prefabs such as AR Camera, Image Target etc. into the scene according to need, delete the original Main Camera object, drag and drop the corresponding augmented contents to be sub-objects of Image Target and adjust the world coordinates and scaling, so that when the target image is identified the augmented content will be registered under the definition of the relative position of the scene, set the appropriate lighting to heighten the atmosphere, finally configurate the parameters and write or modify C \# script to complete the desired function, some parameters shown in Fig. 1 


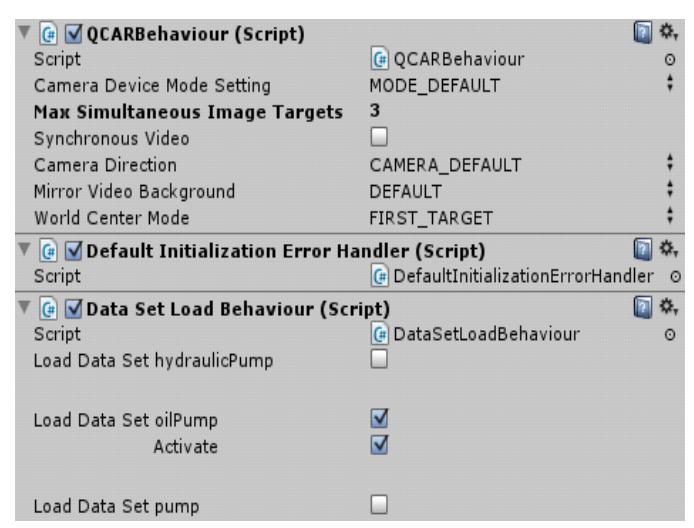

Fig 1. Some important parameters configuration

The system uses Cult3D as interactive software, first installed three program of Cult3D on computer ,then selected the export command in file menu in 3DS MAX, and derived type as C3d , In this way , the file format of the model and animation can be identified by Cult3D Designer . The C3d files are Added to the Cult3D Desiner with appropriate interaction, then output into a . Co file format, the specific steps are as follows:

First of all, in the file add Cult3D designer file menu by introducing just manyou.c3d file. When the Scene Graph ( scene ) window will appear in3ds Max in the production of all models of objects, such as cameras, each view, all the parts, materials and so on, can also be here to be motion the same object component, method is in need of group of an arbitrary object right-click on the mouse, by new dummy generates a a virtual object, the object will be grouped into one.

Step1. Artificial given basketball video segmentation, capture panoramic camera video similar to figure 2 period of $20 \mathrm{~s}$, and all of them as experiment frame sequence image frames;

Step2. To regional segmentation of experimental frames used way, keep the middle area.

Step3. Calculate all the frames used in HSV color components in the space the sum of the histogram $H_{\text {sum }}$.

$$
H_{\text {sum }}=\sum_{i=1}^{n} H\left(I_{i}\right)
$$

$\mathrm{n}$ is the total number of image frames in the 20s video.

Step4. Calculate the sum of all pixels Total $_{\text {sum }}$ in the $H_{\text {sum }}$.

$$
\text { Total }_{\text {sum }}=\sum_{i=1}^{k} H_{\text {sum }}
$$

$\mathrm{k}$ is for image frames color quantization level. Here we will attune (original h component of HSV space for grade 360) quantitative level of 40 , namely $k=40$. The experiment proved that after the quantitative stadium has good color clustering.

Step5. Calculate the average color.

$$
\overline{c o l \_s e q}=\frac{\sum_{i=1}^{n}\left(\operatorname{col}_{\text {max }_{i}}\left(\text { Total }_{\text {sum }}\right)\right)}{n}
$$

$\mathrm{col}_{\text {max }_{i}}\left(\right.$ Total $\left._{\text {sum }}\right)$ is the $\mathrm{i}$ in the value of the corresponding color in the Total ${ }_{\text {sum }}$, the main color sequence number is 5 , so $n=5$,the largest of the five values corresponding to the average value in the Total $_{\text {sum }}$.

\section{Conclusion}

To sum up, the significance of applied research on virtual reality technology in the field of landscape architectural design teaching is enormous. It not only will promote the change in design thinking of the students and the optimization of the design effect, but also will benefit to the 
improvement of teaching effectiveness.

\section{Acknowledgment}

The research was supported by the Science-Technology Foundation for Young Scientist of Hubei Province, China (Grants \#Q20131101), Excellent Young Teachers Program of Wuhan University of Science and Technology (Grants \#2012XZ020), and Social Science Research Foundation for Young Scientist of Hubei Province, China(Grants \#14Q020).

\section{Reference}

[1] Xinghua Song, Bert Jüttler. Modeling and 3D object reconstruction by implicitly defined surfaces with sharp features[J]. Computers \& Graphics. 2012(3)

[2] Rachel McDonnell, Michéal Larkin, Simon Dobbyn, Steven Collins, Carol O’Sullivan. Clone attack! Perception of crowd variety[J]. ACM Transactions on Graphics (TOG). 2012(3)

[3] Soo-Kyun Kim, Chang-Hun Kim. Finding ridges and valleys in a discrete surface using a modified MLS approximation[J]. Computer-Aided Design. 2012(2)

[4] Hong Liu, Mingxi Tang. Evolutionary design in a multi-agent design environment[J]. Applied Soft Computing Journal. 2011(2)

[5] Yutaka Ohtake, Alexander Belyaev, Hans-Peter Seidel. Ridge-valley lines on meshes via implicit surface fitting[J]. ACM Transactions on Graphics (TOG) . 2004 (3) 